\title{
Multilinguales
}

15 | 2021

Recherches algériennes en sociolinguistique et en littérature : discours scientifique/discours militant, quelles frontières?

\section{Recherche en littérature écrite d'expression amazighe : état des lieux et typologie des discours critiques}

Research in written literature of Amazigh expression: state of the art and typology of critical discourses

\section{Salim Ayad}

\section{CpenEdition}

\section{Journals}

Édition électronique

URL : https://journals.openedition.org/multilinguales/6011

DOI : $10.4000 /$ multilinguales.6011

ISSN : 2335-1853

Éditeur

Université Abderrahmane Mira - Bejaia

Référence électronique

Salim Ayad, « Recherche en littérature écrite d'expression amazighe : état des lieux et typologie des discours critiques », Multilinguales [En ligne], 15 | 2021, mis en ligne le 15 juin 2021, consulté le 14

février 2022. URL : http://journals.openedition.org/multilinguales/6011 ; DOI : https://doi.org/10.4000/ multilinguales.6011

Ce document a été généré automatiquement le 14 février 2022.

\section{(i) $\odot$

Multilinguales est mise à disposition selon les termes de la Licence Creative Commons Attribution Pas d'Utilisation Commerciale - Pas de Modification 4.0 International 


\title{
Recherche en littérature écrite d'expression amazighe : état des lieux et typologie des discours critiques
}

\author{
Research in written literature of Amazigh expression: state of the art and \\ typology of critical discourses
}

Salim Ayad

1 Depuis les premiers travaux inaugurés à l'étranger, notamment en France, la recherche dans le domaine de la littérature écrite en tamazight ne cesse de se développer. Les travaux de Chaker $(1987,1992,2006)$ et, ensuite, ceux d'Abrous (1989) ont posé les jalons d'un discours nouveau jusqu'ici inexistant dans le camp des études berbères.

Ce discours tel qu'il est formulé à ces débuts se veut, essentiellement, un regard sur l'ensemble des facteurs socio-historiques ayant contribué à l'émergence d'une littérature écrite, considérée comme l'aboutissement d'une dynamique culturelle enclenchée depuis le début du vingtième siècle par les instituteurs kabyles (la chaîne dite des culturalistes), qui ont donné à la langue, à la littérature, et à la culture berbère un nouvel essor (Chaker, 1987 :14). Il se veut également un regard sur les propriétés linguistiques des textes produits dans cette littérature, caractérisées par les calques lexicaux et sémantiques. Mais aussi par des néologismes (Abrous, 1989: 81), ainsi que des problèmes de la transcription de cette littérature, puisque le berbère manquait d'un système d'écriture.

3 Quelques temps après, toujours en France, d'autres travaux viennent consolider cette tradition «naissante ». Le mémoire de Maitrise d'Ibrahim (1997) portant sur la vie et l'œuvre de Belaid At Ali' ${ }^{1}$, 'inscrivent dans la ligne droite des réflexions amorcées par ces prédécesseurs, mais ce discours critique s'est confirmé avec les DEA de Belgasmia (2001) et celui d'Ameziane (2002). A partir de là, les études sur la littérature écrite ont pris un nouvel essor. Elles se sont détachées d'une manière remarquable des premières 
réflexions. Car, même si l'esprit de leurs prédécesseurs est présent dans leurs travaux, étant leurs réflexions s'inscrivent dans la perspective du processus du "passage à l'écrit » (Chaker, 1992 :2), ces deux études ont eu le mérite d'aborder le contenu des œuvres écrites en kabyle. En effet, les deux auteurs se sont attelés à montrer les propriétés internes des œuvres en décrivant les procédés d'écriture, le redéploiement des genres oraux dans des textes modernes et les différences de styles entre les textes oraux et les textes écrits.

4 Ces mêmes réflexions ont été approfondies par Ameziane $(2004,2006)$ et, ensuite, dans sa thèse de doctorat (2008), dans laquelle il a examiné un corpus plus vaste en adoptant des méthodes de la critique littéraire telles que l'intertextualité et la mythocritique. Ainsi, ce discours prend enfin forme et explore des horizons jusqu'ici ignorés par le discours critique ayant pour objet la littérature écrite.

Ce regard furtif que nous venons de jeter sur les prémices de ce discours n'est, en réalité, que d'ordre indicatif. Car ces travaux nécessitent à eux seuls, tenant compte des conditions dans lesquelles ont vu le jour et leur intérêt dans la constitution d'un champ d'études nouveau, une étude particulière. Cependant, en Algérie, avec les changements qui se sont produits concernant la condition du tamazight dès le début des années 1990 jusqu'aux évolutions récentes, les choses ont beaucoup évolué dans les aspects touchant à la création écrite et aux études littéraires.

Dès lors, la relative effervescence qu'a connu la production littéraire écrite ${ }^{2}$ a constitué un véritable champ d'investissement pour les chercheurs, et il suffit de jeter un coup d'œil sur les travaux réalisés dans ce domaine pour constater l'engouement de la recherche à ce nouveau champ d'étude. Désormais, des recherches de tous types ont abordé cette littérature: mémoires de magistère, articles scientifiques, thèses de doctorat. Soulignons de passage que des dizaines de mémoires de fin d'études de licence ancien régime et master, qui instituent à une critique littéraire universitaire, ont été réalisés dans les différents départements de Langue et Culture Amazighes. Tandis que le discours critique se consolide, au fur et à mesure que cette veine littéraire évolue, ses orientations se sont diversifiées en multipliant les méthodes et les corpus étudiés. Mais la question qui nous préoccupe ici est la suivante: Qu'on est-il de ce discours et de ses orientations? Quelles lectures peut-on en tirer de ces recherches?

7 Tel qu'il se présente aujourd'hui, ce discours se constitue, en partie, par le recours aux différentes méthodes de la critique littéraire, ce qui a permis d'inscrire les problématiques des recherches dans des cadres théoriques stricts. Les unes s'intéressent au contexte des œuvres et le cheminement historique de leur réception, d'autres s'attaquent aux contenus des œuvres, à leurs structures narratives, et d'autres inscrivent cette littérature dans sa relation à la littérature orale. Comme il y a d'autres qui portent sur les thèmes et l'imaginaire ou encore sur la classification générique des œuvres que cette littérature a produites.

8 Mais avant d'aller plus loin dans notre propos, des précisions concernant cette notion de discours critique s'imposent. Dans notre cas, ici considéré, par discours critique nous entendons l'ensemble des discours théoriques ayant pour objet l'œuvre littéraire et plus généralement la littérature. À cet égard, les réflexions esquissées par Todorov (2002) et Compagnon (2002), semblent répondre suffisamment à nos besoins dans cette étude. En effet, dans leurs tentatives de circonscrire les grandes orientations du discours sur la littérature Todorov et Compagnon, chacun de son côté, ont proposé des typologies des grandes tendances qu'ont connu les études littéraires au $20^{\mathrm{em}}$ siècle. 
Todorov emploie le terme de poétique pour désigner les discours sur la littérature et les œuvres, au sein duquel il distingue deux grands discours, bien qu'ils soient complémentaires, l'un porte sur les ouvres et l'autre porte sur l'objet littérature. C'est ce que nous pouvons lire dans ce commentaire :

Dès sa naissance, ce discours n'est pas un, quant à sa finalité et ses formes, mais prend deux directions différentes: ce sont l'exégèse et la théorie. [...]Le discours exégétique emprunte depuis les origines deux chemins séparés: d'une part, l'exégèse littérale, qui consiste à élucider le sens de tel mot incompréhensible, de fournir des références à telle allusion, d'expliquer une telle construction syntaxique; de l'autre, l'exégèse allégorique, qui cherche un sens autre à un texte (ou à un segment de texte) qui en a déjà un. [...]L'objet de la théorie littéraire, en revanche, change radicalement d'une époque à une autre [...]. (Todorov, $2001: 601$ ).

Chez Compagnon, on trouve une autre catégorisation de ce discours. D'ailleurs, lui, à la place de poétique il emploie l'expression critique littéraire pour désigner l'ensemble des méthodes des études littéraires. A ce propos, il écrit :

L'expression « critique littéraire » recouvre aujourd'hui deux activités relativement autonomes. Elle désigne d'une part les comptes rendus de livres dans la presse, à la radio, à la télévision : parlons ici de «critique journalistique ». Elle renvoie d'autre part au savoir sur la littérature, aux études littéraires: parlons cette fois de «critique universitaire » ou « didactique ». (Compagnon, 2001: 430).

De notre côté, c'est à la critique dite universitaire que nous nous intéressons ici, en commençant d'abord par délimiter son objet. La critique universitaire se veut une critique scientifique et érudite, à l'université, disait Compagnon

« on fait de la recherche sur la littérature, on décrit, on analyse, on interprète, et

l'on dépend des jugements littéraires des autres, ou de soi-même comme autre ».

(op, cit : 431).

11 Dans cette dernière, les études littéraires sont regroupées en cinq grands modèles que nous résumons comme suit :

Les modèles contextuels ou explicatifs : dans ce modèle sont classées la sociologie littéraire, la philologie, l'histoire littéraire, la sociologie et la psychanalyse de la littérature, la psychocritique ;

Les modèles profonds ou interprétatifs : où sont rangées la critique biographique, la critique créatrice, la critique des thèmes, de la conscience et des profondeurs;

Le modèle existentialiste : La critique existentialiste a préservé les notions d'individu et de subjectivité promues par les écrivains eux-mêmes ;

Les modèles textuels ou analytiques : où sont classées la linguistique saussurienne, le formalisme russe, le New Criticism, le structuralisme, la sémiotique, la poétique et la narratologie, la stylistique et la tropologie ;

Les modèles "gnostiques » ou indéterminés : l'esthétique de la réception (lecture), la déconstruction (pluralité des sens), le dialogisme et l'intertextualité, le féminisme, le matérialisme culturel ;

En réalité, des répartitions comme celles proposées par Todorov et Compagnon fusent de partout. Beaucoup d'ouvrages traitent de cette classification, dans le but de baliser les grandes orientations des études littéraires, sans pour autant réussir à dessiner des frontières nettes entre les unes et les autres. Ce qu'il y a à retenir de cette aporie est que des consensus autour d'une classification sont loin d'être trouvés, et tant que les études littéraires évoluent on trouvera davantage de désaccords, mais aussi de points communs. Mais pour des raisons méthodologiques et dans le but de cerner les grandes orientations prises par les études sur la littérature écrite amazighe, nous retiendrons la 
classification de Compagnon, et nous comptons nous en inspirer afin de dégager notre propre typologie.

Il n'est certes pas de mon propos d'examiner l'ensemble des paramètres qui font de ce texte un prisme de l'entière condition socio-historique ayant pris part à l'émergence de ce discours. Les principaux d'entre eux ont été mis au jour par la critique : rapport à la langue, impact culturel, revendication identitaire. Autant de traits constitutifs qui, incontournables dans une recherche comme la nôtre, ne me retiendront pas ici. Néanmoins, cette contribution s'attachera à passer en revue l'ensemble des études ayant pour objet la littérature écrite d'expression amazighe. Cet examen tel qu'il se décline aujourd'hui s'appuie, principalement, sur les intitulés et les problématiques traitées dans chaque travail. Une fois décrites et commentées, ces critiques seront regroupées en catégories.

\section{La littérature écrite en tamazight et sa critique}

Dans ce qui suivra, nous nous consacrerons à exposer l'essentiel de ce que la critique a produit comme discours dans le domaine de la littérature écrite d'expression amazighe. À cet effet, on notera que certains travaux, par souci purement méthodologique, seront exclus, par contre nous comptons les inclure dans la bibliographie de cette étude afin de donner une meilleure visibilité sur l'ensemble des travaux réalisés dans ce domaine. Pour une meilleure lecture, nous avons préféré procéder par domaine d'études afin de pouvoir inscrire chacune d'entre elles selon le cadre théorique dans lequel elle s'inscrit.

\subsection{Critique sociologique et histoire littéraire}

Sous cette appellation sont regroupés les travaux ayant pour problématique la littérature écrite dans sa relation à la société. La préoccupation majeure des auteurs de ces travaux est les conditions d'existence de cette littérature telles que les problèmes d'édition et de diffusion, le problème de la nomenclature du nouveau système générique, ainsi que l'effet de la situation sociolinguistique sur la littérature kabyle et le profil des écrivains de cette veine littéraire. Cette orientation de la recherche a été amorcée par Salhi $(2006,2008,2014)$.

16 Le regard de Salhi porte sur les problèmes que rencontre l'édition du roman amazigh et le problème de l'appellation Ungal ("Roman») et l'impact de la situation sociolinguistique et de l'espace littéraire algérien sur les œuvres écrites en kabyle, en revanche l'intérêt de Chemakh (2010) s'oriente vers les conditions dans lesquelles les auteurs de la nouvelle veine littéraire produisent leurs œuvres. Il a cherché, plus précisément, à comprendre comment des écrivains qui n'ont reçu aucune scolarisation dans leur langue maternelle ont acquis les compétences linguistiques et littéraires nécessaires à toute création littéraire. Il confirme que certains écrivains ont appris à écrire en dehors de l'institution scolaire, par des apprentissages individuels et d'autres auteurs tels A. Mezdad, S. Sadi ont suivi les cours que donnait M. Mammeri à l'Université d'Alger jusqu'à ce que ces derniers soient interdits en 1973. En dehors de ces cours un bon nombre d'auteurs, selon les propos de Chemakh, ont appris à écrire le kabyle en caractères latins sans suivre le moindre enseignement du kabyle.

17 Salhi et Sadi (2016) ont présenté un état des lieux du roman d'expression berbère. Ils ont examiné des romans écrits aussi bien en berbère marocain (chleuh et rifain) qu'en 
berbère algérien (kabyle). Ils ont dressé ce panorama sous trois angles différents. Le premier aborde les conditions d'émergence de l'écriture romanesque en berbère en l'incluant dans le grand "champ littéraire maghrébin » et "par rapport aux romans d'expression arabe et d'expression française et en caractérisant les motivations qui l'ont favorisé ». Dans cet axe, il a été question des conditions sociopolitiques et culturelles dans lesquelles évolue le roman d'expression berbère. Le second est consacré au traitement du corpus qui compose le genre romanesque en berbère en tentant de situer ses conditions éditoriales. La troisième et dernière partie expose les grandes tendances thématiques.

Ce regard sociologique sur la littérature écrite, on aura remarqué, a beaucoup évolué dans le temps. Au cours des dernières années, il a pris une autre direction, qui est celle de l'histoire littéraire. C'est dans cette perspective que s'inscrit la thèse de Bellal (2020) qui a essayé par le biais de l'étude des caractéristiques de l'écriture de Belaid At Ali de retracer l'histoire de sa réception.

\subsection{Critique sémiotico-narrative}

19 Les travaux de recherches qu'on peut ranger dans cette catégorie se sont intéressés, particulièrement, à la description des différentes techniques narratives employées par les auteurs des œuvres écrites. Le point commun de ces travaux c'est qu'ils s'inscrivent tous dans le même cadre théorique à savoir la narratologie et la sémiotique du récit développées, notamment, par Genette et Greimas. C'est ce qu'on peut constater chez Berdous (2001), Achili (2002, 2015), Hacid (2007) et Bourai (2007).

D'autres études s'inspirant des approches narratologiques et sémiotiques du texte littéraire, mettent l'accent sur des aspects non traités jusque-là. C'est ce que nous constatons dans l'étude de Boudia (2012), de deux nouvelles de Mezdad extraites du recueil Tuyalin (=«le retour »). L'objet de cette étude a porté sur la narration dans les deux récits, l'espace et le temps ainsi que les personnages. Il a aussi abordé la thématique des deux nouvelles dans leurs liens à la réalité vécue par l'auteur et sa société. Bellal dans son magistère (2012) a étudié trois romans de Amer Mezdad : I wass ( = « Le jour et la nuit »), Tagrest uryu ( =« Hiver brûlant »), et Ass-nni ( = "ce jourlà). C'est une recherche qui porte, à la fois, sur le rapport entre les personnages le contenu textuel des trois romans, ainsi que la signification que procure chaque personnage à chaque texte. Il a aussi abordé les personnages des trois romans dans leurs liens à la réalité vécue par l'auteur et sa société.

Une tentative d'analyse sémiotique des trois romans de Mezdad, Achili (2013), a montré les différentes situations conflictuelles dans lesquelles se trouvent les personnages. Par cette approche, elle a essayé de dégager la signification de chaque situation en confrontant les personnages aux objets. À travers cette analyse, l'auteur explique la relation des œuvres à leur ancrage sociopolitique et culturel.

\subsection{Critique intertextuelle}

L'étude de la filiation qu'il y a entre les œuvres littéraires relevant de celle de la veine écrite et celles de l'oralité a fait l'objet d'étude d'un certain nombre de travaux universitaires qui ont été menés au cours des deux dernières décennies. La problématique qui a été posée par ces travaux consiste à décrire la manière dont ces 
auteurs ont repris et retravaillé des textes traditionnels pour en faire de nouvelles œuvres écrites.

À ce titre, l'étude de Titouche (2002) est inaugurale. Partant d'un point de vue stylistique, il a montré les différents procédés d'écriture entrepris par Belaid At Ali sur des textes traditionnels. Il a dégagé les aspects stylistiques sur lesquels s'est axé le travail de l'auteur. Pour adapter ces récits à l'écrit, Belaid At Ali opère des digressions internes, où il s'étale sur des détails, qui servent à expliquer des épisodes peu explicites dans le texte d'origine. Les récits de Belaid At Ali font aussi recours aux proverbes et aux structures syntaxiques du français ainsi qu'aux emprunts lexicaux à l'arabe et au français.

Pour désigner les textes transférés de l'oralité à l'écrit, dans le processus qu'on appelle "passage à l'écrit », Salhi (2002, 2004), utilise le terme de "délocalisation» afin d'appréhender la relation entre la littérature écrite et la littérature orale. Il distingue cinq types de délocalisation: graphique, linguistique, stylistique, générique et architextuelle. Suivant ces types de délocalisation, établis par Salhi, les textes traditionnels retravaillés par les auteurs de la littérature écrite peuvent faire l'objet de ces cinq types de délocalisation, mais les plus importantes en sont la délocalisation stylistique et générique.

Salhi parle, ailleurs, de la présence de la poésie de Si Mohand chez les poètes contemporains, il écrit à ce propos :

Évoquer, citer et rendre hommage à Si Mohand ou Mohand ici, nombrables sont les évocations dans la poésie écrite et (chantée). Dans les poèmes on évoque le personnage de Si Mohand, soit comme caution de réussite poétique soit comme force langagière capable d'élucider les situations inextricables (Salhi, 2007 :274).

Dans une étude de magistère, Ayad (2008), sur deux textes de Bouamara, en partant du point de vue intertextuel, a dégagé certaines récurrences dans l'appropriation des œuvres de l'oralité dans les nouvelles de Bouamara tirgara et taqsit $n$ Eziz $d$ Ezuzu. En plus de ces deux textes, qui constituent l'ossature des versions recrées par Bouamara, il a énuméré une multitude d'éléments de l'oralité qui appartiennent à des registres et à des catégories diverses du répertoire littéraire orale kabyle.

L'étude de Mohand-Saidi (2011), s'est proposé de comparer les niveaux narratif, descriptif et discursif dans trois variantes du conte tafunast igujilen («la vache des orphelins ») avec celle écrite par Belaid At Ali. L'objectif est de tirer au clair l'apport de Belaid At Ali dans la réappropriation d'un conte traditionnel et de vérifier les différents niveaux sur lesquels se manifeste sa touche. Ceci est dans le but de situer le renouvellement littéraire dans le texte et son impact sur la catégorie générique du texte.

27 Une autre étude, qu'on peut regrouper sous cette perspective, est celle de Medjadi Djejjiga. Dans son magistère (2012), elle a abordé le lien qu'entretient un conte contemporain avec le conte kabyle traditionnel, en étudiant Imețti $n$ Bab idurar d'Akli Kebaili. L'objet principal de ce travail est de déterminer les aspects du renouvellement dans le conte contemporain et de mesurer le degré de fidélité au conte traditionnel.

Dans cette même orientation Ayad et Aissou (2020), ont abordé la poétique du proverbe dans le roman Ihulfan ("les sentiments») de Kaysa Khalifi. Il a été question de commenter le traitement que réserve l'écriture romanesque au proverbe et de montrer son importance dans la constitution du sens globale de l'œuvre. Les réflexions autour de l'intertextualité et son apport dans l'émergence d'une littérature écrite en 
tamazight a été l'œuvre de Ayad (2020). En effet, dans sa thèse il a tenté de montrer le lien entre le recours à l'intertextualité dans l'élaboration des œuvres écrites et les conditions dans lesquelles ces mêmes œuvres ont vu le jour. Il a constaté que l'intertextualité dans ce cas est adoptée, par les auteurs de cette veine littéraire, comme une stratégie d'émergence.

\subsection{Critique thématique} moins nombreuses. En effet, Hamane (1999), dans son étude sur Merwas deg lberğ n yițij ( = « Merwas au château du soleil »), en partant d'un double postulat : structuraliste et thématique, a traité la problématique du déracinement. Celui de la parole et celui relatif à l'espace à travers l'analyse des personnages et de leurs discours.

Abrous (2006), en s'appuyant sur ses recherches précédentes, en tire une conclusion intéressante. Elle remarque que sur quatre romans, bien qu'ils aient des structures spécifiques, leur écriture présente des ressemblances profondes : deux traits d'écriture les unissent. Le premier est d'ordre thématique qui se manifeste dans la question identitaire, déboires de l'émigration, et l'amour d'une terre ingrate. Le second trait est leur ancrage profond dans la symbolique berbère, qui a alimenté l'imaginaire des écrivains et a constitué un socle pour leur écriture. À partir de l'analyse de ces différents éléments certaines conclusions sont dégagées dont: le thème de l'éclatement, qui prend des formes différentes, est central dans Asfel («le sacrifice»), car il mène à la mort. Par contre, dans les trois autres romans, l'éclatement n'est pas synonyme de la mort. Ils sont construits sur la logique de la lutte.

31 Concernant cette orientation de la critique littéraire, nous citerons l'étude de Sadi (2012). Le point de départ de cette étude est la récurrence du thème de l'identité dans les productions romanesques d'expression kabyle. Dans ce cas de figure, le roman Tafrara («l'aurore») présente un cas typique. L'auteur a tenté de répondre à des questions non abordées jusqu'ici, comme la relation de la poétique au thème de l'identité, et sa répercutions sur les éléments du récit ou encore l'impact de la signification de l'identité sur la structure du récit. C'est aussi dans ce type de critique qu'on inscrit sa thèse de doctorat (2019). Dans cette dernière elle a abordé la problématique de l'écriture romanesque en kabyle sous l'angle de la sociopoétique afin d'interroger l'identité même d'une écriture jusqu'ici méconnue de cette littérature.

\section{5. Études des genres}

Cette autre orientation qu'ont prise les études sur la littérature écrite est inaugurée par Rachid Titouche et Zerar Sabrina (2009), les deux auteurs ont essayé une classification du texte Lwali $n$ wedrar («le saint homme de la montagne ») de Belaid At Ali. Ils jugent que ce récit est innovant, car il se distingue nettement de tout récit connu jusqu'alors. Et en recourant aux grilles d'analyse "universelles », disaient-ils, et nonobstant la polémique inhérente à la classification des récits en genres, ils ont tenté dans cet article d'apporter la preuve que ce récit se classait dans la catégorie littéraire «nouvelle » à travers l'étude de l'espace, les personnages et les actions. Notons, sous cette appellation, l'étude de Benallaoua (2012) qui a tenté de dégager une typologie du roman d'expression amazighe. Pour se faire, elle a examiné les éléments du paratexte, 
notamment les titres des romans qui expriment le souci des auteurs de ce genre littéraire à présenter non seulement l'œuvre en elle-même, mais aussi à présenter son genre.

La même problématique est posée autrement, par Ameziane et Salhi (2014), dans une étude consacrée entièrement au genre dénommé tullist (« nouvelle »), considéré comme un genre en "construction ", en discutant le corpus qui le compose. La préoccupation principale est de répondre à la question suivante : qu'est-ce qui compose donc le corpus de cette catégorie de textes appelée tullist? L'importance de cette question, affirmaient-ils est qu'elle se pose comme une condition méthodologique essentielle de la suite et de l'orientation qu'ils veulent donner à l'étude de tullist. Elle est déterminante, aussi bien, de l'évolution de ce qu'on pourrait considérer comme «le genre de la nouvelle » en kabyle, des types de textes qui s'y insèrent que des tendances poétiques que l'analyse est appelée à y définir.

Quelles conclusions tirer de cet exposé ? Si l'on se réfère aux orientations qu'a pris la critique de la littérature écrite en tamazight et à la classification des méthodes de la critique littéraire dressée par Compagnon, une certaine typologie se dégage, et nous la décrivons comme suit :

- un point de vue sociologique inscrit cette littérature dans sa relation à la société et aux conditions dans lesquelles elle est née.

- un point de vue sémiotico-narratif tente de la saisir dans sa structure, son organisation narrative, et les significations qu'entretient une structure d'un texte avec son contenu.

- un point de vue thématique qui tente de traiter des aspects relatifs à l'imaginaire ou aux contenus idéologiques traités par certains auteurs.

- un point de vue intertextuel qui tente de la cerner dans sa relation à la littérature traditionnelle orale et/ou transcrite.

-5.étude des genres, un point de vue selon lequel on a tenté de décrire et/ou de commenter les caractéristiques de quelques genres écrits ainsi que des tentatives de classement.

Voilà en gros comment s'est construit autour de la littérature écrite tout un discours critique qui tente de la circonscrire dans ses différents aspects. Mais quelle lecture pouvons-nous faire à ce parti pris de la recherche dans ce domaine? Qu'y a-t-il d'important à retenir dans notre cas précis? Ce sont des questions qui seront traitées dans la suite de cette contribution et auxquelles nous tenterons d'apporter quelques éléments de réponse.

\section{Regard sur le discours critique}

S'il y a des lectures qui se dégagent à partir de l'examen de l'état de la recherche sur la littérature écrite d'expression amazighe, exposé plus haut, nous retiendrons, en premier lieu, l'intérêt grandissant de la part des chercheurs à cette littérature : elle est lue, commentée et étudiée. Bien que des objections peuvent être formulées à l'égard d'un tel ou tel point de vue, quoi qu'il en soit, cette recherche a donné naissance à un corps critique qui institue, selon les termes de Compagnon, une critique universitaire qui ne cesse de s'affirmer et de s'autonomiser des à priori idéologiques.

Inscrire cette littérature, longtemps minorée ou tout simplement marginalisée, dans les études universitaires revient à lui accorder une légitimité. Dans ce sens, le discours critique joue un rôle déterminant dans sa reconnaissance et par le large public et par la 
communauté scientifique. Dans ce cas précis, le corps critique, en l'absence d'organes officiels, joue, selon Bourdieu (1991), un rôle d'instances de consécration et de légitimation. Puisqu'elle n'est pas seulement étudiée, mais, aussi, elle est enseignée. Et ainsi, elle a conquis de nouveaux territoires, et a acquis un statut qui ne cesse de s'affirmer.

Un deuxième trait qui se dégage de cet état des lieux est que cette littérature est réfléchie comme une interaction entre elle et son ainée, la traditionnelle : bon nombre de travaux ont inscrit leurs réflexions dans cette problématique qui a tendance à montrer le lien indéfectible que tissent les œuvres écrites avec les genres relevant de la tradition orale. Cependant, ces œuvres ne sont pas perçues comme de simples créations, mais on leur fait jouer le rôle de réservoir des savoirs ancestraux, retravaillés par la poétique moderne et présenter sous de nouvelles formes à un public-récepteur soucieux de redécouvrir sa filiation littéraire, linguistique et esthétique. De ce point de vue, cette littérature, du moins d'après notre recherche bibliographique, est essentiellement intertextuelle. À cela, on peut ajouter les points de vue qui tentent de saisir la valeur esthétique, mais surtout symbolique, des créations écrites en kabyle. La présence d'éléments relevant de la culture, de la mythologie est vue comme étant une tentative d'ancrer cette littérature dans sa dimension identitaire berbère. Il est de même pour certaines analyses qui n'ont pas manqué de soulever l'aspect revendicatif de cette littérature. Cette revendication, tissée comme une toile de fond, est une caractéristique majeure des premières œuvres. Aujourd'hui, nul ne peut nier la situation défavorable dans laquelle s'est retrouvé le tamazight au lendemain de l'indépendance de l'Algérie en 1962. Si au départ la revendication pour la reconnaissance du tamazight a pris une dimension purement linguistique, les choses ont évolué avec le temps pour gagner d'autres domaines, et la littérature écrite était l'une des facettes de cette revendication, où elle a joué un rôle déterminant dans la survie de la langue et de la culture amazighes.

Une troisième lecture possible de cet état des lieux est que la réflexion sur les genres nouveaux a constitué un point nodal de ces études. Les tentatives de classement des productions écrites dans des grilles génériques imposent inéluctablement une réflexion, d'une manière particulière, sur la nouvelle configuration de la littérature d'expression kabyle et, d'une manière générale, sur la configuration de la littérature d'expression amazighe. Qu'y a-t-il à ajouter au terme de cette étude? Pour ne pas conclure commençons par rappeler les grands moments de cette contribution. L'objectif que nous nous sommes assigné dans cette réflexion est de rendre compte, un tant soit peu, de la situation de la recherche, en Algérie, dans le domaine de la littérature écrite d'expression amazighe. Pour ce faire, nous avons réuni ici l'essentiel des travaux ayant produit des discours critiques sur cette littérature, tout en tentant, dans le but de dégager une typologie, de les classer selon les grandes tendances que ces discours ont pris. Mais avant, nous avons jugé utile de retracer, à titre indicatif, l'historique de ce discours critique afin de mieux nous situer par rapport à notre problématique.

Ensuite, nous avons tenté une lecture globale de l'ensemble des critiques exposées dans cette étude. Cette lecture, a permis une vue d'ensemble de ce qui a été fait, mais aussi d'en saisir les grandes tendances de ce discours. Mais cela ne s'est pas fait sans difficulté, car nous avons dû, en cours de route, nous séparer de quelques travaux pour éviter de reproduire les mêmes constats. Une autre difficulté à laquelle nous avions dû 
faire face est de dresser un état des lieux des plus fidèles possible. Avouons-nous que cela comporte des risques: les lectures que nous avons proposées restent, tout de même, lacunaires, car nous étions contraints d'opérer des choix sur le contenu à retenir de telle ou telle étude. Nous avons délibérément évacué certaines études : Les cahiers de Belaid, regard sur une cuvre pionnière (2016), les écrits de Belaid At Ali 1909-1950, un auteur et une ceuvre à [r]elire(2016), car nous estimons que Bellal (2019) en a déjà fait un état des lieux des plus détaillés. Nous sommes conscients du fait qu'il est impossible de décrire ce processus dans sa totalité: conditions de l'émergence des discours critiques, leurs tendances et leurs limites, ainsi que les représentations qu'on en fait de ce discours. Autant de questions qui restent en suspend qui nécessiteraient, dans le futur, une meilleure prise en charge de la part des chercheurs afin de déterminer d'une façon détaillée tous les postulats de ce discours et d'en débattre des contenus qu'il véhicule avec plus de précisions.

\section{BIBLIOGRAPHIE}

ABROUS D., La production romanesque kabyle : une expérience de passage à l'écrit, D.E.A, SALEM CHAKER (dir(s)), Aix-en-Provence, Marseille, France, 1989.

ABROUS D., « Eclatement et enracinement dans la production romanesque kabyle », Etudes littéraires africaines : Littérature berbère, $n^{\circ}$ 21, l'Harmattan, Paris, France, 2006, pp 29-39. ACHILI F., «L'intertextualité dans le discours romanesque Kabyle à travers le roman d'Amar Mezdad « Ị̇ d wass », Iles d Imesli nº 3, Tizi Ouzou, 2011, pp 81-94.

ACHILI F., « Dualité de la révolutionnarisation et du changement dans le discours romanesque kabyle A travers la trilogie d'Amar MEZDAD », Iles d Imesli nº 6, Tizi Ouzou, 2014, pp 266-278.

AIT OUALI N., L'écriture romanesque kabyle d'expression berbère de 1946 à 2014, L'Odyssée, Tizi Ouzou, 2015.

AMEZIANE A., Les formes littéraires traditionnelles dans le roman kabyle : du genre aux procédés, D.E.A, ABDELLAH BOUNFOUR (dir(s)), Inalco, Paris, France, 2002.

AMEZIANE A., « La néo-littérature kabyle et ses rapports à la littérature traditionnelle », Etudes littéraires africaines : Littérature berbère, $n^{0}$ 21, l'Harmattan, Paris, France, 2006, pp 20-28.

AMEZIANE A., Tradition et renouvellement dans la littérature kabyle, Thèse de Doctorat, ABDELLAH BOUNFOUR (dir (s.)), Inalco, Paris, 2008.

AMEZIANE, A., SALHI, M A., Tullist kabyle : réflexions préliminaires sur le corpus, dans 3eme Colloque international, Université de Bouira, 2013. Disponible sur : [http://hdl.handle.net/ 123456789/2057], Consulté le 03/03/2021.

AYAD S., Intertextualité et littérature kabyle contemporaine : le cas de Nekni d weyid de Kamal Bouamara, Mémoire de Magistère, CLAUDE FINTZ (dir (s.)), Université de Bejaia, 2008.

AYAD S., AISSOU O., Timmedyezt n yinzi deg wungal Ihulfan n Kaysa Xalifi, Aleph [En ligne], 7 (2) | 2020. Disponible sur : [https://aleph-alger2.edinum.org/2690], Consulté le 22 décembre 2020 
AYAD S., Littérature écrite d'expression kabyle : stratégies et conditions d'émergence, Thèse de Doctorat, KAMEl BOUAMARA (dir (s.)), Université de Tizi Ouzou, 2020.

BELGASMIA E., Tradition orale, oralité et écriture dans les romans de Rachid Aliche, D.E.A, ABDELLAH BOUNFOUR (dir (s.)), Inalco, Paris, 2001.

BELLAL H., Les écrits de Belaid At Ali face à la tradition littéraire kabyle, Thèse de Doctorat, MOHAND AKLI SALI (dir (s.)), Université de Tizi Ouzou, 2020.

BELLAL N., Etude du personnage, en tant que catégorie textuelle, dans les romans kabyles d'Amer Mezdad, Mémoire de Magistère, KAmel Bouamara (dir (s.)), Université de Béjaïa, 2012.

BENALLAOUA A., Contribution à l'étude typologique du roman d'expression kabyle, Mémoire de Magistère, KAMEL BOUAMARA (dir (s.)), Université de Béjaïa, 2012.

BOUDIA A., Contribution à l'analyse textuelle d'un corpus de nouvelles d'expression kabyle, Mémoire de Magistère KAMEL BouAMARA (dir (s.)), Université de Béjaïa, 2012. Bourai o., Asfel : étude narrative et discursive, Mémoire de Magistère, RABAH KAHLOUCHE (dir (s.)), Université de TiziOuzou, 2007.

BOURDIEU P. « Le champ littéraire », In Actes de la recherche en sciences sociales. vol. 89, septembre 1991, pp. 3-46.

Cahiers de Belaid, regard sur une œuvre pionnière, AMEZIANE AMER (dir (s.)), Tira, Béjaia, 2016.

CHAKER S. «L'affirmation identitaire berbère à partir de 1900. Constantes et mutations (Kabylie) », Revue de l'Occident musulman et de la Méditerranée, $n^{\circ} 44$. Berbères, une identité en construction, 1987, pp. 13-34. Disponible sur : [https://doi.org/10.3406/remmm.1987.2152], Consulté le 21/04/2018.

CHAKER S., « La naissance d'une littérature écrite : le cas berbère (Kabylie) », in Bulletin des Etudes Africaines : IX (17/18), Inalco, Paris, 1992, pp 1-7.

CHEMAKH S., « Les conditions de production de la néo-littérature kabyle », Asinag n ${ }^{0}$ 4-5, Rabat, Maroc, 2010, pp 163-168.

COMPAGNON A., «Littéraire, critique », dans Dictionnaire des genres et des notions littéraires, Encyclopeadia Universalis et Albin Michel, 2001, pp 430-448.

Ecrits de Belaid At Ali 1909-1950, un auteur et une œuvre à [r]elire. Actes du colloque international, MOHAND-AKLI SALHI (dir (s).), ENAG, Alger, 2016.

HAMANE F., l'ubiquité du déracinement et son apport dans la rémanence de la littérature algérienne dans Merwas au château du soleil d'Abdellah Hamane, Mémoire de Magistère, FAouzIA SARI (dir(s).), Université d'Oran, 1999.

IBRAHIM M., Vie et œuvre de Belaïd At-Ali, l'auteur des Cahiers de Belaïd ou la Kabylie d'antan, Mémoire de Maîtrise, ABDELLAH BOUNFoUR et SALEM CHAKER (dir (s.)), Inalco, 1997.

MEDJADI J., Le renouvellement du conte kabyle : le cas d'Imețti n Bab idurar d'Akli Kbaili, Mémoire de Magistère, MOHAND-AKLI SALHI (dir (s).), Université de Tizi Ouzou, 2012.

MOHAND SAIDI S., Le récit Tafunast igujilen de Bélaïd Aït Ali : du conte à la nouvelle, Mémoire de Magistère, DJELLALoui MHEMmED (dir (s).), Université de Tizi-Ouzou, 2011.

SADI N., L'expression de l'identité dans le roman tafrara de Salem Zenia, Mémoire de Magistère, DJELlaloui MHEMmed (dir (s).), Université de Tizi Ouzou, 2012. 
SADI N., Problématique de l'écriture romanesque en kabyle, Thèse de Doctorat, MOHAND-AKLI SALHI (dir (s).), Université de Tizi Ouzou, 2019.

SALHI M.A., « La délocalisation des textes oraux. Le cas de deux textes kabyles : Aheddad l-lqalus et taqsit $\mathrm{n}$ Aziz d Âzuzu, dans Echanges et mutations des modèles littéraires entre Europe et Algérie, Tome 2, CHARLES BONN (dir (s).), Paris, L'Harmattan, 2004, pp 205-211.

SALHI M.A., « La nouvelle littérature kabyle et ses rapports à l'oralité traditionnelle », dans La Littérature Amazighe : oralité et écriture, spécificités et perspectives. Actes du colloque international, KICH AZIZ (dir (s.)), Rabat, 2004, pp 103-121.

SALHI M.A., « Regard sur les conditions d'existence du roman kabyle », Studi Magrebini, nuova serie, volume IV, Napoli, 2006, pp 121-127.

SALHI M.A., "Quelques effets de la situation sociolinguistique algérienne sur la littérature kabyle, le berbère en contact », Berber studies, volume 22, 2008, pp 165-173.

SALHI M.A., « l'esprit du poète Si Mohand ou Mhand et la poésie kabyle d'aujourd'hui », in Etudes et Documents Berbères, $\mathrm{N}^{0}$ 25-26, 2007, pp 273-283.

SALHI M.A., « Quelle grille d'analyse pour le (sous) champ littéraire Kabyle? » dans Champs littéraire et stratégies d'écrivains, Les ouvrages du CRASC, 2014, pp 145-154.

SALHI M.A., SADI N, « Le Roman Maghrébin En Berbère ", Contemporary French and Francophone Studies, 20 :1, 2016, pp 27-36. DOI : 10.1080/17409292.2016.1120548.

TITOUCHE R., Les Cahiers de Belaid At Ali : du conte à la nouvelle, Mémoire de Magistère RICHE B (dir(s)) la direction), Université de Tizi-Ouzou, 2002.

TITOUCHE R., ZERAR S, « Naissance du genre 'nouvelle' en tamazight (kabyle) », El-Tawassol n 23 Janvier 2009, pp 65-65.

TODOROV T., " poétique », dans Dictionnaire des genres et des notions littéraires, Encyclopeadia Universalis et Albin Michel, 2001, pp 601-605.

أشيلي ف. ، تحليل الخطاب السردي في رواية الليل و النهار لعمر مزداد، عبد الحميد بورايو(إشراف )،

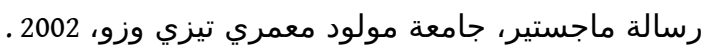

بردوس ن.، السرد في النثر القصصي القبائلي - دراسة مقارنة بين السرد في الحكاية الشعبية

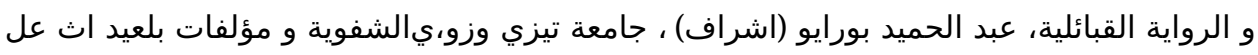
2001.

،.لعمر مزداد، عبد الحميد بورايو(إشراف )، رسالة ماجستير، جامعة تيزي وزوId d wass . حسيد البنية النصية في رواية

\section{NOTES}

1. L'œuvre de Belaid At Ali, Les cahiers de Belaid ou la Kabylie d'antan, marque le début effectif de l'écrit littéraire en kabyle. Elle a été écrite au milieu des années 1940, mais elle n'a été publiée, à titre posthume, par les Pères Dallet et Degezelle dans le Fichier de Documentation Berbère qu'en 1963.

2. En dépit des changements institutionnels et constitutionnels dont la littérature écrite a su en tirer profit, sa condition socio-politique est restée, d'une manière générale, défavorable à plus d'un égard: problèmes d'éditions et de diffusion, le manque de soutien financier, et surtout un 
manque cruel en lectorat. À l'heure actuelle, les choses sur certains plans vont, tout de même, beaucoup mieux, et cette littérature commence à avoir une assise plus solide.

\section{RÉSUMÉS}

Cette contribution essaie de répondre à la question : où on est la recherche dans le domaine de la littérature écrite d'expression amazighe en Algérie ? Les réflexions qui seront développées ici, en nous appuyant sur des propositions théoriques, cherchent à rendre compte de ses orientations et à proposer une typologie des discours critiques produits par la recherche littéraire.

This contribution tries to answer the question: What is the state of the art concerning the research in the field of written literature of Amazigh expression in Algeria? The reflections that will be developed here, based on theoretical proposals, seek to account for its orientations and to propose a typology of the critical discourses produced by literary research.

INDEX

Keywords : written literature, literary criticism, speeches, typology, literary legitimation Mots-clés : littérature écrite, critique littéraire, discours, typologie, légitimation littéraire

\section{AUTEUR}

\section{SALIM AYAD}

Département de Langue et Culture Amazighes, Bejaia, CRLCA, Bejaia, Algérie 